\title{
Asymptotic equilibrium of integro-differential equations with infinite delay
}

\author{
Le Anh Minh ${ }^{1}$ - Dang Dinh Chau ${ }^{2}$
}

Received: 25 June 2014 / Accepted: 2 September 2015/Published online: 21 September 2015

(C) The Author(s) 2015. This article is published with open access at Springerlink.com

\begin{abstract}
The asymptotic equilibrium problems of ordinary differential equations in a Banach space have been considered by several authors. In this paper, we investigate the asymptotic equilibrium of the integro-differential equations with infinite delay in a Hilbert space.
\end{abstract}

Keywords Asymptotic equilibrium · Integro-differential equations · Infinite delay

\section{Introduction}

The asymptotic equilibrium problems of ordinary differential equations in a Banach space have been considered by several authors, Mitchell and Mitchell [3], Bay et al. [1], but the results for the asymptotic equilibrium of integrodifferential equations with infinite delay still is not presented. In this paper, we extend the results in [1] to a class of integro-differential equations with infinite delay in a Hilbert space $H$ which has the following form:

Le Anh Minh

leanhminh@hdu.edu.vn

Dang Dinh Chau

chaudida@gmail.com

1 Department of Mathematical Analysis, Hong Duc University, Thanh Hóa, Vietnam

2 Department of Mathematics, Hanoi University of Science, VNU, Hanoi, Vietnam

$$
\begin{cases}\frac{\mathrm{d} x(t)}{\mathrm{d} t}=A(t)\left(x(t)+\int_{-\infty}^{t} k(t-\theta) x(\theta) \mathrm{d} \theta\right), & t \geqslant 0, \\ x(t)=\varphi(t), & t \leqslant 0\end{cases}
$$

where $A(t): H \rightarrow H, \varphi$ in the phase space $\mathscr{B}$, and $x_{t}$ is defined as

$$
x_{t}(\theta)=x(t+\theta), \quad-\infty<\theta \leqslant 0 .
$$

\section{Preliminaries}

We assume that the phase space $\left(\mathscr{B},\|.\|_{\mathscr{B}}\right)$ is a seminormed linear space of functions mapping $(-\infty, 0]$ into $H$ satisfying the following fundamental axioms (we refer reader to [2])

$\left(\mathrm{A}_{1}\right) \quad$ For $a>0$, if $x$ is a function mapping $(-\infty, a]$ into $H$, such that $x \in \mathscr{B}$ and $x$ is continuous on $[0, a]$, then for every $t \in[0, a]$ the following conditions hold:

(i) $\quad x_{t}$ belongs to $\mathscr{B}$;

(ii) $\quad\|x(t)\| \leqslant G|| x_{t} \|_{\mathscr{B}}$;

(iii) $\left\|x_{t}\right\|_{\mathscr{B}} \leqslant K(t) \sup _{s \in[0, t]}\|x(s)\|+M(t)\left\|x_{0}\right\|_{\mathscr{B}}$

where $G$ is a possitive constant, $K, M:[0, \infty) \rightarrow[0, \infty)$, $K$ is continuous, $M$ is locally bounded, and they are independent of $x$.

$\left(\mathrm{A}_{2}\right) \quad$ For the function $x$ in $\left(A_{1}\right), x_{t}$ is a $\mathscr{B}$-valued continuous function for $t$ in $[0, a]$.

$\left(\mathrm{A}_{3}\right) \quad$ The space $\mathscr{B}$ is complete. 


\section{Example 1}

(i) Let $B C$ be the space of all bounded continuous functions from $(-\infty, 0]$ to $H$, we define $C^{0}:=$ $\left\{\varphi \in B C: \lim _{\theta \rightarrow-\infty} \varphi(\theta)=0\right\}$ and $C^{\infty}:=\{\varphi \in$ $B C: \lim _{\theta \rightarrow-\infty} \varphi(\theta)$ exists in $\left.H\right\}$ endowed with the norm

$$
\|\varphi\|_{\mathscr{B}}=\sup _{\theta \in(-\infty, 0]}\|\varphi(\theta)\|
$$

then $C^{0}, C^{\infty}$ satisfies $\left(\mathrm{A}_{1}\right)-\left(\mathrm{A}_{3}\right)$. However, $B C$ satisfies $\left(A_{1}\right)$ and $\left(A_{3}\right)$, but $\left(A_{2}\right)$ is not satisfied.

(ii) For any real constant $\gamma$, we define the functional spaces $C_{\gamma}$ by

$C_{\gamma}=\left\{\varphi \in C((-\infty, 0], X): \lim _{\theta \rightarrow-\infty} e^{\gamma \theta} \varphi(\theta)\right.$ exists in $\left.H\right\}$

endowed with the norm

$$
\|\varphi\|_{\mathscr{B}}=\sup _{\theta \in(-\infty, 0]} e^{\gamma \theta}\|\varphi(\theta)\| .
$$

Then conditions $\left(\mathrm{A}_{1}\right)-\left(\mathrm{A}_{3}\right)$ are satisfied in $C_{\gamma}$.

Remark 1 In this paper, we use the following acceptable hypotheses on $K(t), M(t)$ in $\left(A_{1}\right)($ iii) which were introduced by Hale and Kato [2] to estimate solutions as $t \rightarrow \infty$,

$\left(\gamma_{1}\right) K=K(t)$ is a constant for all $t \geqslant 0$;

$\left(\gamma_{2}\right) M(t) \leqslant M$ for all $t \geqslant 0$ and some $M$.

Example 2 For the functional space $C_{\gamma}$ in Example 1, the hypotheses $\left(\gamma_{1}\right)$ and $\left(\gamma_{2}\right)$ are satisfied if $\gamma \geqslant 0$.

Definition 1 Equation (1) has an asymptotic equilibrium if every solution of it has a finite limit at infinity and, for every $h_{0} \in H$, there exists a solution $x(t)$ of it such that $x(t) \rightarrow h_{0}$ as $t \rightarrow \infty$.

\section{Main results}

Now, we consider the asymptotic equilibrium of Eq. (1) which satisfies the following assumptions:

$\left(\mathrm{M}_{1}\right) \quad A(t)$ is a strongly continuous bounded linear operator for each $t \in \mathbb{R}^{+}$;

$\left(\mathrm{M}_{2}\right) \quad A(t)$ is a self-adjoint operator for each $t \in \mathbb{R}^{+}$;

$\left(\mathrm{M}_{3}\right) \quad k$ satisfies

$$
\int_{0}^{+\infty}|k(\theta)| \mathrm{d} \theta=L<+\infty \text {; }
$$

$\left(\mathrm{M}_{4}\right) \quad$ There exists a constant $T>0$ such that

$$
\sup _{h \in S(0,1)} \int_{T}^{\infty}\|A(t) h\| \mathrm{d} t<q<\frac{1}{\kappa},
$$

herein $S(0,1)$ is a unit ball in $H, \kappa=L(K+M)+$ 1 , where $K, M, L$ are given in $\left(\gamma_{1}\right),\left(\gamma_{2}\right)$ and $\left(M_{3}\right)$.

Theorem 1 If $\left(\mathrm{M}_{1}\right),\left(\mathrm{M}_{2}\right),\left(\mathrm{M}_{3}\right)$ and $\left(\mathrm{M}_{4}\right)$ are satisfied, then Eq. (1) has an asymptotic equilibrium.

Proof We shall begin with showing that all solutions of (1) has a finite limit at infinity. Indeed, Eq. (1) may be rewritten as

$\frac{\mathrm{d} x(t)}{\mathrm{d} t}=A(t)\left(x(t)+\int_{-\infty}^{0} k(-\theta) x_{t}(\theta) \mathrm{d} \theta\right)$,

then for $t \geqslant s \geqslant T$ we have

$$
x(t)=x(s)+\int_{s}^{t} A(\tau)\left(x(\tau)+\int_{-\infty}^{0} k(-\theta) x_{\tau}(\theta) \mathrm{d} \theta\right) \mathrm{d} \tau
$$

and

$$
\begin{aligned}
& \|x(t)\| \\
& =\sup _{h \in S(0,1)}\left|\left\langle x(s)+\int_{s}^{t} A(\tau)\left(x(\tau)+\int_{-\infty}^{0} k(-\theta) x_{\tau}(\theta) \mathrm{d} \theta\right) \mathrm{d} \tau, h\right\rangle\right| \\
& \leqslant\|x(s)\|+\sup _{h \in S(0,1)} \int_{s}^{t}\left|\left\langle x(\tau)+\int_{-\infty}^{0} k(-\theta) x_{\tau}(\theta) \mathrm{d} \theta, A(\tau) h\right\rangle\right| d \tau \\
& \leqslant\|x(s)\|+q\left((L K+1) \sup _{\xi \in[0, t]}\|x(\xi)\|+L M\|\varphi\|_{\mathscr{B}}\right)
\end{aligned}
$$

implies

$$
\left|\left\|x ( t ) \left|\||\leqslant| x(s)\|+q\left((L K+1)\||| x(t)\| \mid+L M\|\varphi\|_{\mathscr{B}}\right)\right.\right.\right.
$$

or

$$
|\|x(t)\|| \leqslant \frac{\|x(s)\|+q L M\|\varphi\|_{\mathscr{B}}}{1-q(L K+1)}
$$

where

$$
\|x(t)\| \mid=\sup _{0 \leqslant \xi \leqslant t}\|x(\xi)\| .
$$


Now, we conclude that $x(t)$ is bounded since

$0<q<\frac{1}{\kappa}=\frac{1}{L(K+M)+1}<\frac{1}{L K+1} \Rightarrow q(L K+1)<1$

and by (4).

Putting

$M^{*}=\sup _{t \in \mathbb{B}}\|x(t)\|$,

we have

$$
\begin{aligned}
& \|x(t)-x(s)\|=\sup _{h \in S(0,1)}|<x(t)-x(s), h>| \\
& \leqslant \sup _{h \in S(0,1)} \int_{s}^{t}\left|<A(\tau)\left(x(\tau)+\int_{-\infty}^{0} k(-\theta) x_{\tau}(\theta) \mathrm{d} \theta\right), h>\right| \mathrm{d} \tau, \\
& \leqslant\left[M^{*}(L K+1)+L M\|\varphi\|_{\mathscr{B}}\right] \sup _{h \in S(0,1)} \int_{s}^{t}\|A(\tau) h\| d \tau \rightarrow 0
\end{aligned}
$$

as $t \geqslant s \rightarrow+\infty$. That means all solutions of (1) have a finite limit at infinity. To complete the proof, it remains to show that for any $h_{0} \in H$, there exists a solution $x(t)$ of (1) such that

$\lim _{t \rightarrow+\infty} x(t)=h_{0}$.

Indeed, let $h_{0}$ be an arbitrary fixed element of $H$; we choose the initial function $\varphi$ belongs to $\mathscr{B}$ such that $\varphi(0)=h_{0}$ and $\|\varphi\|_{\mathscr{B}} \leqslant\left\|h_{0}\right\|$ and consider the functional

$$
\begin{aligned}
g_{1}(t, h)= & \left\langle h_{0}, h\right\rangle \\
& -\int_{t}^{\infty}\left\langle A(\tau)\left(h_{0}+\int_{-\infty}^{\tau} k(\tau-\theta) x_{0}(\theta) \mathrm{d} \theta\right), h\right\rangle \mathrm{d} \tau
\end{aligned}
$$

We have

$$
\begin{aligned}
\left|g_{1}(t, h)\right| & \leqslant\left\|h_{0}\right\|\|h\|+\int_{t}^{+\infty} \| x_{0}(\tau) \\
& +\int_{-\infty}^{\tau} k(\tau-\theta) x_{0}(\theta) \mathrm{d} \theta\|\| A(\tau) h \| \mathrm{d} \tau .
\end{aligned}
$$

Since $x_{0}(\tau) \equiv h_{0}$, then

$$
\left|g_{1}(t, h)\right| \leqslant \| h_{0}||(\|h\|+q \kappa) .
$$

It follows from Riesz representation theorem that there exists an element $x_{1}(t)$ in $H$, such that

$g_{1}(t, h)=\left\langle x_{1}(t), h\right\rangle$ and

$$
\left\|x_{1}(t)\right\| \leqslant\left\|h_{0}\right\|(1+q \kappa) .
$$

Now, we consider the functional

$$
\begin{aligned}
& g_{2}(t, h)=\left\langle h_{0}, h\right\rangle \\
& \quad-\int_{t}^{+\infty}\left\langle A(\tau)\left(x_{1}(t)+\int_{-\infty}^{\tau} k(\tau-\theta) x_{1}(\theta) \mathrm{d} \theta\right), h\right\rangle \mathrm{d} \tau .
\end{aligned}
$$

By an argument analogous to the previous one, we get

$$
\left|g_{2}(t, h)\right| \leqslant \| h_{0}||\left[|| h \|+q \kappa+(q \kappa)^{2}\right]
$$

and there exists an element $x_{2}(t)$ in $H$, such that

$g_{2}(t, h)=\left\langle x_{2}(t), h\right\rangle$

with

$$
\left\|x_{2}(t)\right\| \leqslant\left\|h_{0}\right\|\left(1+q \kappa+(q \kappa)^{2}\right) .
$$

Continuing this process, we obtain the linear continuous functional

$$
\begin{aligned}
& g_{n}(t, h)=\left\langle h_{0}, h\right\rangle \\
& \quad-\int_{t}^{+\infty}\left\langle A(\tau)\left(x_{n-1}(t)+\int_{-\infty}^{\tau} k(\tau-\theta) x_{n-1}(\theta) \mathrm{d} \theta\right), h\right\rangle \mathrm{d} \tau
\end{aligned}
$$

and $x_{n}(t) \in H$ such that

$$
g_{n}(t, h)=\left\langle x_{n}(t), h\right\rangle
$$

satisfies the following estimate

$$
\left\|x_{n}(t)\right\| \leqslant\left(1+q \kappa+(q \kappa)^{2}+\cdots+(q \kappa)^{n}\right)\left\|h_{0}\right\| \leqslant \frac{\left\|h_{0}\right\|}{1-q \kappa} .
$$

Futhermore,

$$
\left\|x_{n}(t)-x_{n-1}(t)\right\| \leqslant\left\|h_{0}\right\|(q \kappa)^{n} .
$$

This inequality shows that $\left\{x_{n}(t)\right\}$ is uniformly convergent on $[T,+\infty)$ since $q \kappa<1$. Put

$$
x(t)=\lim _{n \rightarrow+\infty} x_{n}(t) .
$$

In (5), let $n \rightarrow+\infty$, we have

$$
\begin{aligned}
\langle x(t), h\rangle= & \left\langle h_{0}, h\right\rangle \\
& -\int_{t}^{+\infty}\left\langle A(\tau)\left(x(t)+\int_{-\infty}^{\tau} k(\tau-\theta) x(\theta) \mathrm{d} \theta\right), h\right\rangle \mathrm{d} \tau
\end{aligned}
$$

and since 


$$
\begin{aligned}
& \left|\left\langle x_{n}(t), h_{0}\right\rangle\right|<\int_{T}^{+\infty} \| x_{n-1}(\tau) \\
& +\int_{-\infty}^{\tau} k(\tau-\theta) x_{n-1}(\theta) \mathrm{d} \theta\|\| A(\tau) h \| \mathrm{d} \tau
\end{aligned}
$$

or

$\left|\left\langle x_{n}(t), h_{0}\right\rangle\right| \leqslant \frac{\left\|h_{0}\right\| q}{1-q \kappa}$,

we have $x_{n}(t) \rightarrow h_{0}$ as $q \rightarrow 0$, which means that there exists a solution of (1) converging to $h_{0}$. The theorem is proved.

Open Access This article is distributed under the terms of the Creative Commons Attribution 4.0 International License (http:// creativecommons.org/licenses/by/4.0/), which permits unrestricted use, distribution, and reproduction in any medium, provided you give appropriate credit to the original author(s) and the source, provide a link to the Creative Commons license, and indicate if changes were made.

\section{References}

1. Bay, N.S., Hoan, N.T., Man, N.M.: On the asymptotic equilibrium and asymptotic equivalence of differential equations in Banach spaces. Ukr. Math. J. 60(5), 716-729 (2008)

2. Hale, J.K., Kato, J.: Phase space for retarded equations with infinite delay. Fukcialaj Ekvacioj 21, 11-41 (1978)

3. Mitchell, A.R., Mitchell, R.W.: Asymptotic equilibrium of ordinary differential systems in a Banach space. Theory Comput. Syst. 9(3), 308-314 (1975) 